\title{
Probióticos e resposta imune
}

\author{
Probiotics and immune response
}

\author{
Mario de Menezes Coppola ${ }^{1}$ Carlos Gil Turnes ${ }^{2}$
}

\section{- REVISÃO BIBLIOGRÁFICA -}

\section{RESUMO}

Os probióticos são bactérias que produzem efeitos benéficos no hospedeiro, usadas para prevenir e tratar doenças, como promotores de crescimento e como imunoestimulantes. Nesta revisão abordam-se as principais aplicações dos probióticos, com ênfase nas informações recentes sobre suas propriedades de modular a resposta imune.

Palavras-chave: probióticos, Saccharomyces boulardii, Bacillus cereus var. toyoi, imunomodulação.

\section{ABSTRACT}

Probiotics are bacteria that produce beneficial effects on their hosts, used in the prevention and treatment of diseases, as growth promoters and immune-modulators. The most important applications of probiotics, with emphasis on their properties to modulate the immune response, are reviewed here.

Key words: probiotics, Saccharomyces boulardii, Bacillus cereus var. toyoi, immune-modulation.

\section{INTRODUÇÃO}

O termo probiótico deriva do grego e significa "pró-vida", sendo o antônimo de antibiótico, que significa "contra a vida". Ao longo do tempo, esta denominação teve diferentes acepções. LILLY \& STILLWEL (1965) a usaram para denominar substâncias secretadas por um protozoário que estimularam o crescimento de outros, e PARKER (1974), para denominar suplementos alimentares destinados a animais, incluindo microrganismos e substâncias que afetam o equilíbrio da microbiota intestinal. FULLER (1989) considerou que os probióticos são suplementos alimentares que contêm bactérias vivas que produzem efeitos benéficos no hospedeiro, favorecendo o equilíbrio de sua microbiota intestinal, entanto HAVENAAR \& HUIS IN'T VELD (1992) consideraram que são culturas únicas ou mistas de microrganismos que, administrados a animais ou humanos, produzem efeitos benéficos no hospedeiro por incremento das propriedades da microbiota nativa. Esses autores restringiram o uso desse termo a produtos que contenham microrganismos viáveis que promovem a saúde de humanos ou animais, e que exercem seus efeitos no aparelho digestivo, no trato respiratório superior ou no trato urogenital (HAVENAAR et al., 1992). SCHREZENMEIR \& DE VRESE (2001) propuseram que o termo probiótico deveria ser usado para designar preparações ou produtos que contêm microrganismos viáveis definidos e em quantidade adequada, que alteram a microbiota própria das mucosas por implantação ou colonização de um sistema do hospedeiro, e que produzem efeitos benéficos em sua saúde.

O termo prebiótico é utilizado, a diferença de probiótico, para designar ingredientes alimentares não digeríveis que beneficiam o hospedeiro por estimular seletivamente o crescimento e/ou a atividade de uma ou um número limitado de espécies bacterianas no cólon (GIBSON \& ROBERFROID, 1995), e o termo simbiótico para designar produtos que contêm probióticos e prebióticos associados. Como a palavra sugere sinergismo, ela deveria ser restringida a produtos em que o componente prebiótico favoreça seletivamente o probiótico (SCHREZENMEIR \& DE VRESE, 2001).

${ }^{1}$ Médico Veterinário, Mestre em Ciências, Centro de Biotecnologia e Faculdade de Veterinária da Universidade Federal de Pelotas (UFPEL). ${ }^{2}$ Médico Veterinário, Doutor em Ciências, Centro de Biotecnologia e Faculdade de Veterinária, UFPel, CP 354, 96010-900, Pelotas, RS., Brasil, E-mail: gil@ufpel.tche.br. Autor para correspondência. 
Vários microrganismos são usados como probióticos, entre eles bactérias ácido- lácticas, bactérias não ácido lácticas e leveduras (Tabela 1). Além das propriedades mencionadas, os probióticos devem ser inócuos, manter-se viáveis por longo tempo durante a estocagem e transporte, tolerar o baixo $\mathrm{pH}$ do suco gástrico e resistir à ação da bile e das secreções pancreática e intestinal, não transportar genes transmissores de resistência a antibióticos e possuir propriedades anti-mutagênicas e anticarcinogênicas, assim como resistir a fagos e ao oxigênio (HAVENAAR et al., 1992; SALMINEN et al., 1998; OUWEHAND et al., 1999; SAARELA et al., 2000; HOLZAPFEL \& SCHILLINGER, 2002).

\section{APLICAÇÕES DOS PROBIÓTICOS}

Os probióticos são usados em medicina humana na prevenção e tratamento de doenças, na regulação da microbiota intestinal, em distúrbios do metabolismo gastrintestinal, como imunomoduladores, e na inibição da carcinogênese. Em medicina veterinária, além dessas aplicações, podem também ser usados como promotores de crescimento, constituindo-se em uma alternativa aos antibióticos, cujo uso indiscriminado pode selecionar cepas resistentes.

\section{MECANISMOS DE AÇÃO DOS PROBIÓTICOS}

O modo de ação dos probióticos não foi ainda completamente esclarecido, embora tenham sido sugeridos vários processos que podem atuar independentemente ou associados. Um deles é a exclusão competitiva, em que o probiótico competiria com os patógenos por sítios de fixação e nutrientes, impedindo sua ação transitoriamente (HAVENAAR et al., 1992; OUWEHAND et al., 1999; CROSS, 2002). A exclusão competitiva explicaria a necessidade da administração continuada e a elevadas doses dos probióticos, para manifestar seus efeitos.

Os probióticos podem também afetar patógenos através da síntese de bacteriocinas (VILLANI et al., 1995; RODRIGUEZ, 1996; NAIDU et al., 1999), de ácidos orgânicos voláteis (AUDISIO et al., 2000; JIN et al. 2000; OGAWA et al., 2001) e de peróxido de hidrogênio (HAVENAAR et al., 1992;

Tabela 1 - Microrganismos com propriedades de probióticos.

\begin{tabular}{|c|c|c|c|}
\hline Lactobacillus & Bifidobacterium & Outras bactérias ácido lácticas & Bactérias não ácido lácticas \\
\hline \multirow[t]{2}{*}{ L. acidophilus } & B. adolescentis & $\begin{array}{l}\text { Enterococcus } \\
\text { faecalis }\end{array}$ & Bacillus cereus var. toyoi \\
\hline & & & Escherichia coli cepa nissle \\
\hline \multirow[t]{2}{*}{ L. amylovorus } & B.animalis & Enterococcus & \\
\hline & & faecium & $\begin{array}{l}\text { Propionibacterium } \\
\text { freudenreichii }\end{array}$ \\
\hline \multirow[t]{2}{*}{ L. casei } & B. bifidum & Lactococcus lactis & \\
\hline & & & Saccharomyces cerevisiae \\
\hline \multirow[t]{2}{*}{ L. crispatus } & B. breve & Leuconstoc mesenteroides & Saccharomyces boulardii \\
\hline & & Pediococcus acidilactici & \\
\hline \multirow[t]{2}{*}{$\begin{array}{l}\text { L. delbrueckii subsp. } \\
\text { bulgaricus }\end{array}$} & B. infantis & & \\
\hline & & Sporolactobacillus inulinus & \\
\hline \multirow[t]{2}{*}{ L. gallinarum } & & & \\
\hline & B. lactis & Streptococcus thermophilus & \\
\hline \multicolumn{4}{|l|}{ L. gasseri } \\
\hline \multicolumn{4}{|l|}{ L. johnssonii } \\
\hline \multicolumn{4}{|l|}{ L. paracasei } \\
\hline \multicolumn{4}{|l|}{ L. plantarum } \\
\hline \multicolumn{4}{|l|}{ L. reuteri } \\
\hline L. rhamnosus & & & \\
\hline
\end{tabular}

Adaptado de HOLZAPFEL et al. (2001). 
NAIDU et al., 1999), ou atuar sobre o metabolismo celular, reduzindo a concentração de amônia no organismo (KOZASA, 1986), e liberando enzimas como a lactase (DE VRESE et al., 2001).

Seus efeitos anticarcinogênicos podem ser atribuídos à inibição de enzimas pro-carcinogênicas ou a estimulação do sistema imunitario do hospedeiro. A administração de Lactobacillus casei foi relacionada com a indução de uma resposta antitumoral mediada por células T e a ativação de macrófagos (KATO et al., 1988), assim como a supressão da formação de tumores de cólon em camundongos (KATO et al., 1994) e a inibição de metástases pulmonares (MATSUZAKI \& YOKOKURA, 1987; MATSUZAKI et al., 1990).

\section{PROBIÓTICOS DE BACILLUS}

Desde 1990, o Centro de Biotecnologia da Universidade Federal de Pelotas desenvolve pesquisas sobre a utilização de probióticos elaborados com Bacillus cereus var. toyoi destinados a animais. A principal vantagem de Bacillus sobre as bactérias ácido lácticas, na elaboração de probióticos, reside em sua capacidade de esporular, o que lhes confere maior sobrevivência durante o trânsito estomacal (HOA et al., 2000), e durante a elaboração, transporte e armazenamento das rações (GIL TURNES et al., 1999). Estes probióticos promovem o ganho de peso e o controle de diarréias, e reduzem a mortalidade perinatal em suínos (ZANI et al., 1998), e em frangos (RICHTER et al., 1999). CUEVAS et al. (2000) comprovaram aumento do ganho de peso e diminuição significativa da mortalidade pela síndrome de ascite em frangos, e LÖHNERT et al. (1999) aumento no ganho de peso de $10 \%$ em terneiros machos suplementados com este probiótico.

Outras cepas de Bacillus foram também utilizadas com resultados satisfatórios como probióticos. B. cereus CIP 5832 produziu efeitos benéficos em porcas durante o fim da prenhês e a lactação, assim como em leitões em crescimento (ALEXOPOULOS et al., 2001).

A eficácia dos probióticos depende da concentração bacteriana na ração. ROTH \& KIRCHGESSNER (1988a) comunicaram que o ganho de peso total e a conversão alimentar de leitões melhoraram quando o probiótico foi fornecido nas concentrações de $5 \times 10^{8}$ ou $1 \times 10^{9}$ esporos viáveis por $\mathrm{Kg}$ de ração, mas não em concentrações menores. EIDELSBURGER et al. (1992) utilizaram o mesmo probiótico na concentração de $2,5 \times 10^{8}$ esporos por $\mathrm{kg}$ de ração, e o ganho de peso e o consumo de ração caíram $8,1 \%$ e $9,0 \%$, respectivamente, enquanto a conversão alimentar aumentou em 5,6\%. KYRIAKIS et al. (1999) relataram que B. licheniformes foi mais efetivo no controle de Escherichia coli enterotoxigênica em leitões na concentração de $1 \times 10^{7}$ esporos por grama de ração que a uma concentração dez vezes menor. Em terneiros, constatou-se também uma relação entre concentração e eficácia, já que o ganho de peso e a eficiência alimentar aumentaram 3,9\% e 3,2\%, respectivamente, quando a concentração de $\boldsymbol{B}$. cereus var. toyoi foi de $1 \times 10^{9}$ esporos viáveis por $\mathrm{kg}$, enquanto a concentrações menores o probiótico não teve efeito (ROTH \& KIRCHGESSNER, 1988b).

Além dos efeitos mencionados, bactérias do gênero Bacillus podem estimular a resposta imune e serem utilizadas como imunomoduladores. B. firmus aumentou a resistência contra a infecção experimental por Listeria monocytogenes em camundongos (MÁRA et al., 1994). BELIAVSKAIA et al. (2001) demonstraram que $\boldsymbol{B}$. subtilis recombinante evitou a imunossupressão causada pelas vacinas replicantes contra Parvovirus e Cinomose, acelerando a formação de clones de memória e aumentando a resposta imune específica devido à ação do interferon $\alpha 2$ secretado pela bactéria no interior do lúmen intestinal. CONCEIÇÃO et al. (2002) demonstraram que o probiótico CenBiot estimulou a resposta imune humoral a uma bacterina de Escherichia coli em camundongos, e COPPOLA et al. (2003) comprovaram que este probiótico aumentou significativamente a resposta imune de camundongos a uma vacina replicante de Parvovirus canino.

\section{PROBIÓTICOS DE SACCHAROMYCES BOULARDII}

Saccharomyces boulardii é uma levedura não patogênica utilizada na prevenção e tratamento de diversas doenças gastrintestinais (LOURENSHATTINGH \& VILJOEN, 2001), que mantêm suas propriedades probióticas ainda quando administrada junto com antimicrobianos (ROLFE, 2000). Probióticos preparados com essa levedura protegeram camundongos das toxinas de Clostridium difficile (CORTHIER et al., 1992), e camundongos convencionais e gnotobióticos contra infecções por Salmonella typhimurium e Shigella flexneri (RODRIGUES et al., 1996). Sua administração aumentou significativamente as concentrações de IgA intestinal em ratos (BUTS et al., 1990), assim como de antitoxina A de $\boldsymbol{C}$. difficile na IgA intestinal (QAMAR et al., 2001). Uma protease extraída da levedura inibiu os efeitos das toxinas A e B de $\boldsymbol{C}$. difficile na mucosa 
do cólon de humanos (CASTAGLIUOLO et al., 1999).

A administração de probiótico elaborado com a levedura favoreceu a resposta imune celular tipos 1 e 2 estimulando a produção de IFN- $\gamma$ e IL-4 em camundongos gnotobióticos (RODRIGUES et al., 2000). COPPOLA et al. (2003) comprovaram que $\boldsymbol{S}$. boulardii aumentou a resposta imune humoral de camundongos a uma bacterina de $\boldsymbol{E}$. coli.

\section{EFEITOS DOS PROBIÓTICOS NA IMUNIDADE}

Profusa bibliografia, a maioria relatando estudos com bactérias ácido-lácticas, demonstra que os probióticos têm efeito imunoestimulante em animais e no homem, apesar de ainda não estarem esclarecidos os mecanismos pelos quais isto ocorre (CROSS, 2002). Esse efeito pode estar relacionado à capacidade de os microrganismos do probiótico interagirem com as placas de Peyer e as células epiteliais intestinais, estimulando as células B produtoras de $\operatorname{IgA}$ e a migração de células T do intestino (PERDIGÓN \& HOLGADO, 2000). Também tem sido demonstrado que os probióticos favorecem a atividade fagocítica inespecífica dos macrófagos alveolares, sugerindo uma ação sistêmica por secreção de mediadores que estimulariam o sistema imune (CROSS, 2002).

MAASEN et al. (2000) comprovaram, no entanto, que a síntese de citocinas pela mucosa intestinal dependia da cepa de Lactobacillus utilizada, salientando a necessidade de realizar uma cuidadosa seleção das cepas candidatas a probiótico. $L$. casei apresentou efeito imunomodulador similar ao de Bacillus Calmette-Guerin (BCG), Corynebacterium parvum e Streptococcus pyogenes (PERDIGÓN \& ALVAREZ, 1992). Este lactobacilo induziu uma resposta celular tipo 1 em camundongos gnotobióticos, aumentando a produção in vitro de IL-12 por células peritoniais e de IFN- $\gamma$ por células esplênicas (NEUMAN et al., 2000). Sua administração protegeu camundongos contra patôgenos intestinais pela indução do aumento da capacidade fagocítica dos macrófagos peritoniais e da atividade das enzimas envolvidas na fagocitose (KATO et al., 1983; PERDIGÓN \& ALVAREZ, 1992), da atividade das células assassinas (natural killer) (KATO et al., 1984), da produção de fator citotóxico pelas células de Kupfer e macrófagos peritoniais (HASHIMOTO et al., 1985), e da secreção de IgA no lúmen intestinal (PERDIGÓN et al., 1991; PERDIGÓN et al., 1995).

$\mathrm{O}$ efeito de outros lactobacilos sobre a imunidade também tem sido estudado. ÁVILA et al. (1998) compararam a eficiência de $\boldsymbol{L}$. acidophilus, de vacina específica, e da associação destes dois produtos no controle da diarréia por $\boldsymbol{E}$. coli enterotoxigênica em leitões, e demonstraram que os melhores resultados foram obtidos com a combinação de vacina e probiótico. Outro lactobacilo, L. casei cepa Shirota, estimulou a resposta imune celular aumentando as concentrações de IFN- $\gamma$, TNF- $\alpha$ e IL12, provocando uma redução nos títulos de vírus da Influenza no trato respiratório quando administrada por via nasal (HORI et al., 2001), e oral (HORI et al., 2002).

L. rhamnosus reduziu a morbidade de $\boldsymbol{E}$. coli 0157: H7 em camundongos infectados experimentalmente, estimulando o aumento da atividade fagocítica e dos títulos de anticorpos específicos na IgA (SHU \& GILL, 2002). PERDIGÓN et al. (1999) demonstraram que L casei e $\boldsymbol{L}$. plantarum interagiram com as células $M$ das placas de Peyer, estimulando a imunidade secretora específica. VITINI et al. (2000) testaram a influência da administração oral de diferentes espécies de bactérias ácido lácticas tais como $\boldsymbol{L}$. casei, $\boldsymbol{L}$. acidophilus, L. rhamnosus, L. delbrueckii subsp. bulgaricus, L. plantarum, Lactococcus lactis e Streptococcus thermophilus, e verificaram que o aumento da produção de IgA nem sempre esteve correlacionado com um aumento no número de células $\mathrm{T} \mathrm{CD}_{4}+$, indicando que algumas bactérias testadas somente induziram uma ativação das células B produtoras de $\operatorname{Ig}$ A. Também foi observado que não houve influência sobre as células $\mathrm{T} \mathrm{CD}_{8}+$, indicando que essas bactérias não foram capazes de induzir citotoxicidade.

Outras bactérias ácido lácticas também apresentaram atividade imuno-moduladora. YASUI et al. (1999) comprovaram que a administração de Bifidobacterium breve estimulou o sistema imune humoral em camundongos provocando aumento da produção de IgA anti-Rotavírus e de IgG antivírus da Influenza, protegendo-os contra essas duas infecções. SHU et al. (2001) relataram que leitões tratados com B. lactis HN109 apresentaram diminuição da diarréia associada com Rotavírus e $\boldsymbol{E}$. coli, concomitante ao aumento dos títulos de anticorpos contra patôgenos específicos no trato gastrintestinal, da concentração de neutrófilos sangüíneos e da resposta proliferativa dos linfócitos $\mathrm{T}$.

Com relação a bactérias não ácido-lácticas, pode citar-se, entre outros, o trabalho de COPPOLA et al. (2003), que estudaram o efeito de probióticos preparados com B. cereus var. toyoi e com S. boulardii na resposta imune de camundongos à vacinação simultânea com uma bacterina tetravalente de $\boldsymbol{E}$. coli patogênica para suínos e uma vacina replicante contra 
a Parvovirose canina. Comprovaram que o probiótico de $\boldsymbol{S}$. boulardii induziu uma resposta humoral significativamente mais alta à bacterina, enquanto o probiótico de B. cereus var. toyoi induziu uma resposta significativamente mais alta à vacina de Parvovirus canino. Foi detectado que esplenocitos de camundongos suplementados com $S$. boulardii produziram IL-4 quando estimuladas com fimbrias purificadas de $\boldsymbol{E}$. coli in vitro.

\section{CONCLUSÕES}

As informações geradas ao longo dos últimos anos indicam que vários probióticos têm, além de sua atividade como promotores de crescimento e reguladores da microbiota das mucosas, efeito imunomodulador, embora a forma de ação seja pouco conhecida. As evidências acumuladas sobre os benefícios decorrentes do uso dos probióticos justificam o aprofundamento dos estudos sobre seu modo de ação, a fim de otimizar sua utilização como profiláticos, promotores de crescimento e imunomoduladores.

\section{REFERÊNCIAS BIBLIOGRÁFICAS}

ALEXOPOULOS, C. et al. Field evaluation of a bioregulator containing live Bacillus cereus spores on health status and performance of sows and their litters. Journal of Veterinary Medicine, Berlin, v.48, n.3, p.137-145, 2001.

AUDISIO, M.C.; OLIVER, G.; APELLA, M.C. Protective effect of Enterococcus faecium J96, a potential probiotic strain, on chicks infected with Salmonella pullorum. Journal of Food Protection, Des Moines, v.63, n.10, p.13331337, 2000.

ÁVILA, F.A. et al. Use of vaccine and probiotic in the control of swine diarrhea caused by enterotoxigenic Escherichia coli. Arquivo Brasileiro de Medicina Veterinária e Zootecnia, Belo Horizonte, v.50, n.5, p.505-511, 1998.

BELIAVSKAIA, V.A. et al. Adjuvant properties of subalin, a recombinant interferon-producing probiotic. Zhurnal Mikrobiologii Epidemiologii i Immunobiologii, Moskva, v.78, n.6, p.77-82, 2001.

BUTS, J.P. et al. Stimulation of secretory $\operatorname{IgA}$ and secretory component of immunoglobulins in small intestine of rats treated with Saccharomyces boulardii. Digestive Diseases and Sciences, New York, v.35,n.2, p.251-256, 1990.

CASTAGLIUOLO, I. et al. Saccharomyces boulardii protease inhibits Clostridium difficile toxin A and B effects in human colonic mucosa. Infection and Immunity, Washington. v.67, n.1, p.302-307, 1999.

CONCEIÇÃO, F.R., ZANI, J.L., GIL-TURNES, C. Effect of probiotic CenBiot on the humoral response to an Escherichia coli bacterin. Food and Agricultural Immunology, Basingstoke, v.14, n.2, p.135-140, 2002.

COPPOLA, M.M., CONCEIÇÃO, F.R., GIL-TURNES, C. Effect of Saccharomyces boulardii and Bacillus cereus var. toyoi on the humoral and cellular response of mice to vaccines. Food and Agricultural Immunology, Basingstoke, v.16, (no prelo) 2004.

CORTHIER, G. et al. Effect of oral Saccharomyces boulardii treatment on the activity of Clostridium difficile toxins in mouse digestive tract. Toxicon, Oxford, v.30, n.12, p.1583-1589, 1992.

CROSS, M.L. Microbes versus microbes: immune signals generated by probiotic lactobacilli and their role in protection against microbial pathogens. FEMS Immunology and Medical Microbiology, Amsterdam, v.34, n.4, p.245-253, 2002.

CUEVAS, A.C. et al. El efecto del Bacillus toyoi sobre el comportamiento productivo en pollos de engorda. Veterinaria México, México, v.31, n.4, p.301-308, 2000.

DE VRESE, M. et al. Probiotics-compensation for lactase insufficiency. American Journal of Clinical Nutrition, Bethesda, v.73, n.2, p.421S-429S, 2001.

EIDELSBURGER U.; KIRCHGESSNER M.; ROTH F. X. Zum einfluss von fumarsaure, salzsaure, natriumformiat, Tylosin und Toyocerin auf tagliche zunahmen, futteraufnahme, futterverwertung und verdaulichkeit. 11. Unterschungen zur nutritiven wirksamkeitvon organischen sauren in der ferkelaufzucht. Journal of Animal Physiology and Animal Nutrition, Berlin, v.68, n.4-5, p.82-92, 1992.

FULLER, R. Probiotics in man and animals. Journal of Applied Bacteriology, Oxford, v.66, n.5, p.365-378, 1989.

GIBSON, G.R.; ROBERFROID, M.B. Dietary modulation of the human colonic microbiota. Introducing the concept of prebiotics. Journal of Nutrition, Bethesda, v.125, n.6, p.1401-1412, 1995.

GIL-TURNES, C. et al. Properties of the Bacillus cereus strain used in probiotic CenBiot. Brazilian Journal of Microbiology, São Paulo, v.30, n.1, p.11-14, 1999.

HASHIMOTO, S. et al. Cytotoxic factor production by Kupfer cells elicited with Lactobacillus casei and Corynebacterium parvum. Cancer Immunology Immunotherapy, New York, v.20, n.2, p.117-121, 1985.

HAVENAAR, R.; HUIS IN'T VELD, M. J.H. Probiotics: a general view. In: WOOD, B.J.B. Lactic acid bacteria in health and disease 1. Amsterdam : Elsevier Applied Science, 1992. p.151170 .

HAVENAAR, R.; BRINK, B.T.; HUIS INT`VELD, J.H.J. Selection of strains for probiotic use. In: FULLER, R. Probiotics: the scientific basis. London : Chapman e Hall, 1992. p.209-224.

HOA, N.T. et al. Characterization of Bacillus species used for oral bacteriotherapy and bacterioprophylaxis of gastrointestinal disorders. Applied and Environmental Microbiology, Washington, v.66, n.12, p.5241-5247, 2000.

HOLZAPFEL, W. H. et al. Taxonomy and important features of probiotic microorganisms in food and nutrition. American Journal of Clinical Nutrition, Bethesda, v.73, n.2, p.365S-373S, 2001. 
HOLZAPFEL, W.H.; SCHILLINGER, U. Introduction to pre and probiotics. Food Research International, Amsterdam, v.35, n.23, p.109-116, 2002.

HORI, T. et al. Effect of intranasal administration of Lactobacillus casei Shirota on Influenza virus infection of upper respiratory tract in mice. Clinical and Diagnostic Laboratory Immunology, New York, v.8, n.3, p.593-597, 2001.

HORI, T. et al. Augmentation of cellular immunity and reduction of Influenza virus titer in aged mice fed Lactobacillus casei strain Shirota. Clinical and Diagnostic Laboratory Immunology, New York, v.9, n.1, p.105-108, 2002.

JIN, L.Z.; MARQUARDT, R.R.; BAIDOO, S.K. Inhibition of enterotoxigenic Escherichia coli K88, K99 and 987P by the Lactobacillus isolates from porcine intestine. Journal of the Science of Food and Agriculture, Sussex, v.80, n.5, p.619-624, 2000.

KATO, I.; YOKOKURA, T.; MUTAI, M. Macrophage activation by Lactobacillus casei in mice. Microbiology and Immunology, Tokyo, v.27, n.7, p.611-618, 1983.

KATO, I.; YOKOKURA, T.; MUTAI, M. Augmentation of mouse natural killer cell activity by Lactobacillus casei and its surface antigens. Microbiology and Immunology, Tokyo, v.28, n.2, p.209-217, 1984.

KATO, I.; YOKOKURA, T.; MUTAI, M. Correlation between increase in Ia-bearing macrophages and induction of $\mathrm{T}$ cell dependent antitumor activity by Lactobacillus casei in mice. Cancer Immunology Immunotherapy, New York, v.26, n.3, p.215221, 1988.

KATO, I.; ENDO, K.; YOKOKURA, T. Effects of oral administration of Lactobacillus casei on antitumor responses induced by tumor resection in mice. International Journal of Immunopharmacology, Oxford, v.16, n.1, p.29-36, 1994.

KOZASA, M. Toyocerin (Bacillus toyoi) as growth promotor for animal feeding. Microbiology Aliments Nutrition, n.4, p.121-135, 1986.

KYRIAKIS, S.C. et al. The effect of probiotic LSP 122 on the control of post-weaning diarrhoea syndrome of piglets. Research in Veterinary Science, Oxford, v.67, n.3, p.223-228, 1999.

LILLY, D.M.; STILLWEL, R.H. Probiotics. Growth promoting factors produced by micro-organisms. Science, Washington, v.147, n.3659, p.747-748, 1965.

LÖHNERT, H.J.; OCHRIMENKO, W.I.; BARGHOLZ, J. Influence of the feed additive "Toyocerin" on the rearing result of calves. In: SYMPOSIUM VITAMINS AND ADDITIVES IN NUTRITION OF MAN AND ANIMAL, 7., 1999, Jena. Abstracts... Jena, Thuringia: Institut für Ernährungswissenschaften- Universität Jena, 1999. p.52.

LOURENS-HATTINGH, A.; VILJOEN, B.C. Growth and survival of a probiotic yeast in dairy products. Food Research International, Amsterdam, v.34, n.9, p.791-796, 2001.

MAASSEN, C.B.M. et al. Strain-dependent induction of cytokine profiles in the gut by orally administered Lactobacillus strains. Vaccine, Oxford, v.18, n.23, p.2613-2623, 2000.

MÁRA, M. et al. Resistance to infection and activation of monocyte-macrophage system caused by Bacillus firmus and its fractions. Folia Microbiologica, Praga, v.39, n.2, p.147-151, 1994.
MATSUZAKI, T.; YOKOKURA, T. Inhibition of tumor metastasis of Lewis lung carcinoma in C57BL/6 mice by intrapleural administration of Lactobacillus casei. Cancer Immunology Immunotherapy, New York, v.25, n.2, p.100-104, 1987.

MATSUZAKI, T.; SHIMIZU, Y.; YOKOKURA, T. Augmentation of antimetastatic effect on Lewis lung carcinoma (3LL) in C57BL/ 6 mice by priming with Lactobacillus casei. Medical Microbiology and Immunology, New York, v.179, n.3, p.161-168, 1990.

NAIDU, A.S.; BIDLACK, W.R.; CLEMENS, R.A. Probiotic spectra of lactic acid bacteria (LAB). Critical Reviews in Food Science and Nutrition, Boca Raton, v.38, n.1, p.13-126, 1999.

NEUMAN, E. et al. Lactobacillus acidophilus UFV-H2B20 induces type 1 responses by cells of gnotobiotic mice. In : THE PROSPECTS OF PROBIOTICS AND THERAPY OF DISEASES OF YOUNG, 2000, High Tatras. Proceedings... High Tatras, Slovak Republic : Department of Gnotobiology and Diseases of YoungResearch Institute of Veterinary Medicine, Kosice, 2000. p.85.

OGAWA, M. et al. Inhibition of in vitro growth of Shiga toxinproducing Escherichia coli $\mathrm{O} 157: \mathrm{H} 7$ by probiotic Lactobacillus strains due to production of lactic acid. International Journal of Food Microbiology, Amsterdam, v.68, n.1-2, p.135-140, 2001.

OUWEHAND, A.C. et al. Probiotics: mechanisms and established effects. International Dairy Journal, Amsterdam, v.9, n.1, p.4352, 1999.

PARKER, R.B. Probiotics, the other half of the antibiotic story. Animal Nutrition Health, n.29, p.4-8, 1974.

PERDIGÓN, G.; ALVAREZ, S.; HOLGADO, A.P.R. Immunoadjuvant activity of oral Lactobacillus casei-influence of dose on the secretory immune-response and protective capacity in intestinal infections. Journal of Dairy Research, New York, v.58, n.4, p.485-496, 1991.

PERDIGÓN, G.; ALVAREZ, S. Probiotics and the immune state. In: FULLER, R. Probiotics: the scientific basis. London : Chapman e Hall, 1992. p.145-180.

PERDIGÓN, G. et al. Immune system stimulation by probiotics. Journal of Dairy Science, Savoy, v.78, n.7, p.1597-1606, 1995.

PERDIGÓN, G. et al. Study of the possible mechanisms involved in the mucosal immune system activation by lactic acid bacteria. Journal of Dairy Science, Savoy, v.82, n.6, p.1108-1114, 1999.

PERDIGÓN, G.; HOLGADO, A.P.R. Mechanisms involved in the immunostimulation by lactic acid bacteria. In: FULLER, R.; PERDIGÓN, G. Probiotics 3: Immunodulation by the Gut Microflora and Probiotics. Dordrecht : Kluwer Academic, 2000. p.213-233.

QAMAR, A. et al. Saccharomyces boulardii stimulates intestinal immunoglobulin A immune response to Clostridium difficile toxin A in mice. Infection and Immunity, Washington, v.69, n.4, p.3762$2765,2001$.

RICHTER, G.; KÜHNE, I.; KÖHLER, H. Test of Toyocerin in broiler fattening. In: SYMPOSIUM VITAMINS AND ADDITIVES IN NUTRITION OF MAN AND ANIMAL, 7., 1999, Jena. Abstracts... Jena, Thuringia : Institut für Ernährungswissenschaften - Universität Jena, 1999. p.52-53. 
RODRIGUES, A.C. et al. Effect of Saccharomyces boulardii against experimental oral infection with Salmonella typhimurium and Shigella flexneri in conventional and gnotobiotic mice. Journal of Applied Bacteriology, Oxford, v.81, n.3, p.251-256, 1996

RODRIGUES, A.C.P. et al. Q. Saccharomyces boulardii induces the production of type 1 and type 2 cytokines in gnotobiotic mice. In : THE PROSPECTS OF PROBIOTICS AND THERAPY OF DISEASES OF YOUNG, 2000, High Tatras. Proceedings... High Tatras, Slovak Republic : Department of Gnotobiology and Diseases of Young-Research Institute of Veterinary Medicine, Kosice, 2000. p.87.

RODRIGUEZ, J.M. Antimicrobial spectrum, structure, properties and mode of action of nisin, a bacteriocin produced by Lactococcus lactis. Food Science and Technology International, New York, v. 2, n.2, p.61-68, 1996

ROLFE, R.D. The role of probiotic cultures in the control of gastrointestinal health. Journal of Nutrition, Bethesda, v.130, n.2, p. $396 \mathrm{~S}-402 \mathrm{~S}, 2000$

ROTH, F.X.; KIRCHGESSNER, M. Nutritive wirksamkeit von toyocerin. 1 Ferkelaufzucht. Landwirtschaftliche-Forschung, Frankfurt, v.41, n.12, p.58-62, 1988a.

ROTH, F.X.; KIRCHGESSNER, M. Nutritive wirksamkeit von toyocerin. 2 Kalbermast. Landwirtschaftliche-Forschung, Frankfurt, v.41, n.1-2, p.63-70, 1988 b.

SAARELA, M. et al. Probiotic bacteria: safety, functional and technological properties. Journal of Biotechnology, Amsterdam, v.84, n.3, p.197-215, 2000
SALMINEN, S.; OUWEHAND, A.C.; ISOLAURI, E. Clinical applications of probiotic bacteria. International Dairy Journal, Amsterdam, v.8, n.5-6, p.563-572, 1998.

SCHREZENMEIR, J.; DE VRESE, M. Probiotics, prebiotics and symbiotics-approaching a definition. American Journal of Clinical Nutrition, Bethesda, v.73, n.2, p.361S-364S, 2001.

SHU, Q.; QU, F.; GILL, H.S. Probiotic treatment using Bifidobacterium lactis HN019 reduces weanling diarrhoea associated with rotavirus and Escherichia coli infection in a piglet model. Journal of Pediatric Gastroenterology and Nutrition, Philadelphia,v.33, n.2, p.171-177, 2001

SHU, Q.; GILL, H.S. Immune protection mediated by the probiotic Lactobacillus rhamnosus HN001 (DR20ä) against Escherichia coli $\mathrm{O} 157$ : $\mathrm{H} 7$ infection in mice. FEMS Immunology and Medical Microbiology, Amsterdam, v.34, n.1, p.59-64, 2002.

VILLANI, F. et al. Antilisterial activity of thermophilin 347, a bacteriocin produced by Streptococcus thermophilus. International Journal of Food Microbiology, Amsterdam, v.25, n.2, p.179-190, 1995

VITINI, E. et al. Gut mucosal immunostimulation by lactic acid bacteria. Biocell, Mendoza, v.24, n.3, p.223-232, 2000.

YASUI, H. et al. Immunomodulatory function of lactic acid bacteria. Antonie Van Leeuwenhoek International Journal of General and Molecular Microbiology, Dordrecht, v.76, n.1, p.383-389, 1999.

ZANI, J.L. et al. Effect of probiotic CenBiot on the control of diarrhoea and feed efficiency in pigs. Journal of Applied Microbiology, Oxford, v.84, n.1, p.68-71, 1998 